\title{
Phase transitions in clusters
}

\author{
R.S. Berry \\ Department of Chemistry, University of Chicago, 5735 South Ellis Avenue, Chicago, IL 60637, USA
}

\section{B.M. Smirnov}

Institute for High Temperatures RAS, 13/19 Izhorskaya Str., Moscow 127412, Russia

E-mail: bmsmirnov@gmail.com

Received December 12, 2008

\begin{abstract}
General concepts of cluster phase transitions are reviewed as well as the cluster behavior near the melting point. Configuration excitation determines the nature of the cluster phase transitions, but a significant contribution to the entropy jump is given by thermal motion of atoms that allows one to characterize the phase transition through thermal atom motion in the Lindemann and other criteria. The phase coexistence near the melting point is the peculiarity of not large clusters. The void concept of phase transitions with a void as an elementary configuration excitation allows one to describe the phase transition for clusters and macroscopic atomic systems. Phase transitions in metal clusters resemble those in clusters with pairwise atomic interactions, but their numerical parameters are other because of a large number of isomers and an additional electron degree of freedom. Cluster models are convenient for the analysis of macroscopic atomic systems. They allow us to understand the nature of glassy transitions and the reason of absence of a stable infinite crystal lattice for gases at zero temperature and high pressure.
\end{abstract}

PACS: 36.40. Ei Phase transitions in clusters;

61.72.J- Point defects and defect clusters;

64.70.D- Solid-liquid transitions;

64.70.P- Glass transitions of specific systems.

Keywords: phase transitions, thermal motion, clusters, macroscopic atomic systems.

\section{Introduction}

According to the thermodynamic definition $[1,2]$, the aggregate state of a macroscopic atomic system is a uniform space distribution of atoms restricted by a boundary, and the phase transition is a transition between two aggregate states. In thermodynamic terms, an aggregate state of a macroscopic system of atoms is described by thermodynamic parameters, as the temperature $T$, the entropy $S$, the internal energy $E$, the free energy $F$, and other thermodynamic potentials. A phenomenological character is a lack of the thermodynamic description, and the universality is its advantage.

Transferring from macroscopic atomic systems to clusters, systems of a finite number of identical atoms, causes a question, is it possible to use for clusters a thermodynamic description with ignoring the fluctuations. Along with the thermodynamic description, clusters admit a dynamic description with the analysis of motion of each atom, while this description is cumbrous. Hence, the analysis of clusters gives the understanding the nature of macroscopic atomic systems whose models are clusters.

Especially, it is of importance in a range of the phase transition. The most striking property distinguishing clusters from bulk matter is their dynamic phase coexistence [3-7]. This means that such systems have a temperature-pressure domain of coexistence, in which, part of the time, the cluster resides in one aggregate state, and the remainder, in the other (in a two-phase domain; there can be other domains with more coexisting phases).

Thus, basing on different methods of the cluster analysis, one can understand various aspects of cluster phase transitions by comparison of different methods. This allows us to understand the nature of phase transitions in clusters and macroscopic atomic systems. 


\section{Types of cluster excitations}

The analysis of the cluster potential energy surface (PES) is used along with the dynamic and thermodynamic cluster descriptions where thermodynamics is applicable if typical cluster times are large compared to times of energy exchange between neighboring cluster atoms. This is fulfilled for clusters that are both microcanonical and canonical atom ensembles, i.e., if a cluster is found under isothermal or adiabatical external conditions. It is of importance for clusters where interaction between nearest neighbors dominates. These clusters have the PES with many local minima [8-13]. In particular, for Lennard-Jones clusters, the number of local minima of the PES rises rapidly with the number $n$ of component atoms, to become of order of a thousand even for $n$ of $13[8,9,14]$. This property of the PES determines a specific cluster behavior at low temperatures $[8,15]$. In the course of evolution a typical time for a cluster to remain near a given local minimum considerably exceeds a typical oscillation time. This allows one to treat the residence in the basin around each local minimum as a specific configurational excitation state, albeit transient. In this way one can separate the oscillation and configurational degrees of freedom of the cluster atoms [16]. This character of cluster evolution is the basis of the understanding of cluster behavior. Then the phase transition may be considered as a result of configuration transitions.

One can simplify the PES concept by introducing the elementary configurational excitation, a void [17] or empty internal space, so that a certain configuration cluster excitation includes the formation of some number of voids in a cluster as it is shown in Fig. 1 for a 13-atom cluster where interaction between nearest neighbors dominate. Then

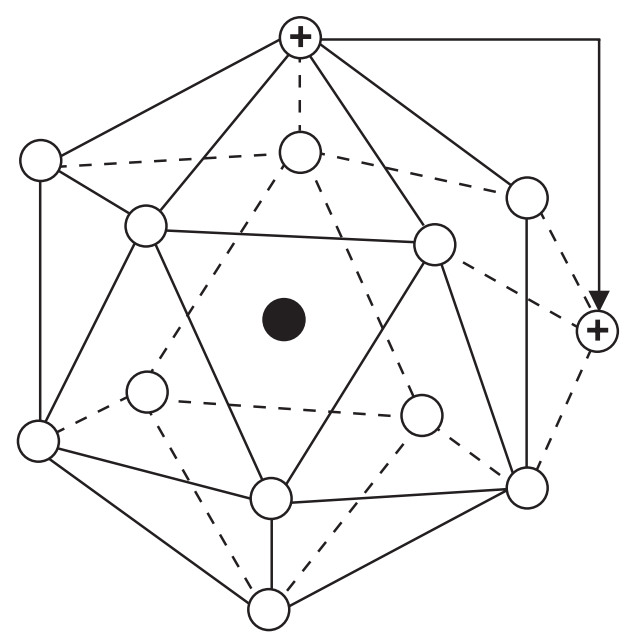

Fig. 1. Configuration excitation of a cluster consisting of 13 atoms where interaction between nearest neighbors dominates. A transferring atom is shown by a cross, and an arrow indicates its transition. configuration excitation results from formation of one void that is a perturbed surface vacancy. In terms of voids, an aggregate cluster state is a group of configuration cluster states which correspond to local minima of the PES with nearby energies. In addition, this combination of configuration excitations must be realized with a remarkable probability (up to one) under some conditions. At a large number of voids, this definition transfers to the definition of an aggregate state of a bulk atomic system. In contrast to macroscopic atomic systems, one elementary excitation is possible for an excited cluster aggregate state as it takes place for a 13 -atom cluster.

\section{Phase transition in 13-atom cluster}

It is convenient to demonstrate the above cluster properties for a 13-atom cluster with dominating interaction between nearest neighbors that includes also the Lennard-Jones cluster. The ground configuration state (the solid aggregate state) corresponds to the icosahedral atom structure, and the first excited configuration state respects to transition of one atom to the cluster surface (Fig. 1). We connect this configuration state with the liquid aggregation state. The latter includes a group of atom configurations that are separated from the ground configuration state by barriers or saddle points, and the energies of these transitions at zero temperature are shown in Fig. 2 [18]. At higher temperatures the barriers between configurationally excited states with one atom transition disappears, and they are combined in one (liquid) aggregate state.

We postulate above that a cluster is located a long time in a certain aggregate state and transfers fast in other state. This is confirmed by the distribution on the total kinetic energy of cluster atoms [3] under adiabatic conditions that has a bimodal form (Fig. 3), so that the cluster is

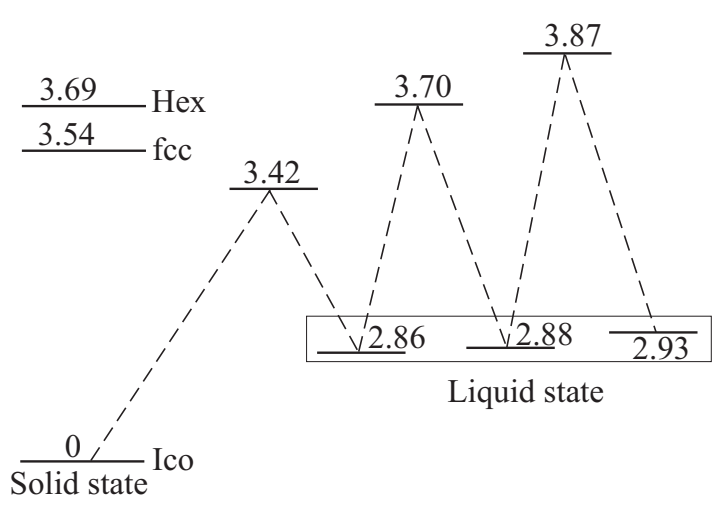

Fig. 2. The excitation and barrier energies for the lowest configuration excitations of the 13-atom Lennard-Jones cluster [18] are expressed in units $D$, the dissociation energy of breaking of one bond. Structures of the solid cluster: Hex - hexagonal, fcc - face-centered cubic, Ico - icosahedral. 

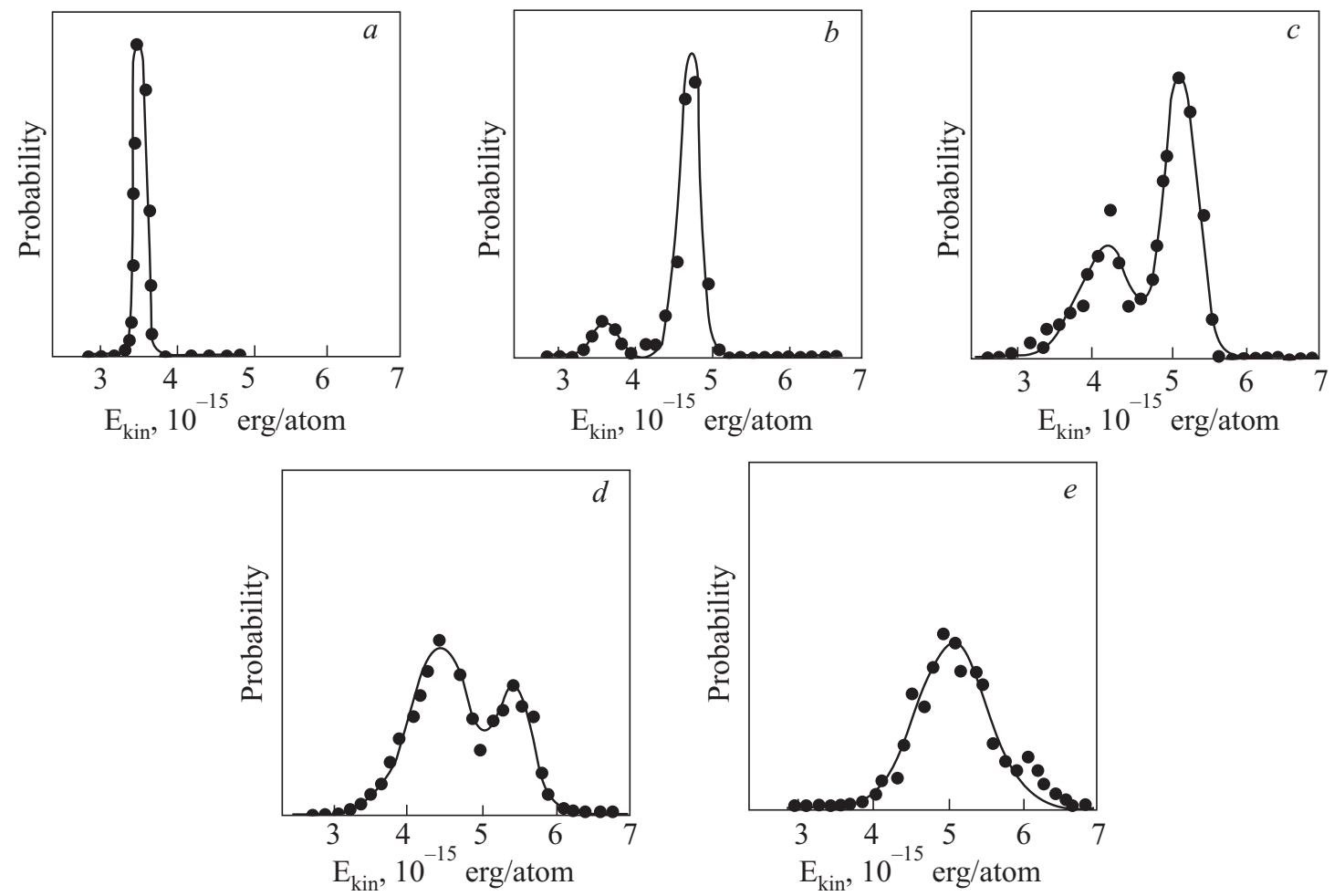

Fig. 3. The distribution function on the total kinetic energy of atoms $E_{\text {kin }}$ for the 13-atom Lennard-Jones cluster at some excitation energies [3] where the energy of breaking of one bond is taken as the unity. The excitation energies $E_{\mathrm{ex}}: 7.59(a), 11.01(b), 12.88$ $(c), 14.13(d), 16.23(e)$.

found in the solid or liquid aggregate state, and the probability of an intermediate state is very small.

This determines the character of phase coexistence that relates to conditions of a microcanonical [3] and canonical [19] atom ensemble. Such a form of phase coexistence together with relatively large times of location in each aggregate state allows one to consider a cluster as a thermodynamic object.

Treating the results of computer simulation with separation of aggregate states, one can obtain various thermodynamic information about a cluster. Note that within the framework of thermodynamic description we neglect fluctuations and the time dependence for the total kinetic energy of cluster atoms has the form Fig. 4, $a$ [15] while a typical real dependence for a cluster as a microcanonical atom ensemble is given in Fig. 4, $b$ [20].

Hence in transition to thermodynamics we average over a large time. Then the average total energy $E$ of cluster atoms that is a sum of the total kinetic energy $K$ of atoms and their total potential energy $U$ on the basis of formula

$$
E=U+K \text {. }
$$

Therefore, defining a configurationally excited aggregate state as a kinetically linked group of states near local minima of PES of similar excitation energies [15,21], we apply formula (1) to the total energies of the solid $E_{\text {sol }}$ and liquid $E_{\text {liq }}$ aggregate states separately, so that this formula takes the form

$$
\begin{aligned}
& E_{\text {sol }}=E_{0}+K_{\text {sol }}+U_{\text {sol }}, \\
& E_{\text {liq }}=E_{0}+\Delta E+K_{\text {liq }}+U_{\text {liq }},
\end{aligned}
$$

where $E_{0}$ is the total energy of atoms in the solid state at zero temperature, $\Delta E$ is the energy of configurational excitation for the liquid state; $K_{\text {sol }}$ and $K_{\text {liq }}$ are the total kinetic energies of atoms for the solid and liquid aggregate states; $U_{\text {sol }}$ and $U_{\text {liq }}$ are the total potential energies of atoms measured from the global minimum atomic configuration. Based on formula (2) and restricted to two aggregate states, we obtain as the parameters to describe the cluster the energy $\Delta E$ for configuration excitation, the temperature of atoms, and the anharmonicity parameter $\eta$ introduced as

$$
\begin{aligned}
& \eta_{\text {sol }}=\frac{K_{\text {sol }}}{K_{\text {sol }}+U_{\text {sol }}}, \\
& \eta_{\text {liq }}=\frac{K_{\text {liq }}}{K_{\text {liq }}+U_{\text {liq }}} .
\end{aligned}
$$

This parameter is introduced separately for the solid and liquid aggregate states. Because the liquid configuration corresponds to a looser atomic structure, $\eta_{\text {liq }}<\eta_{\text {sol }}$, and in the case of harmonic cluster oscillations we obtain $\eta=1 / 2$. Expressing the atom temperature through the total kinetic energy of atoms, we represent formula (2) in the form 


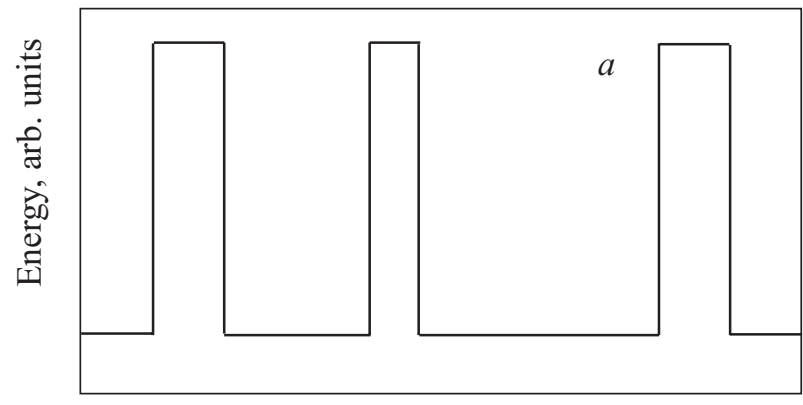

Time, steps

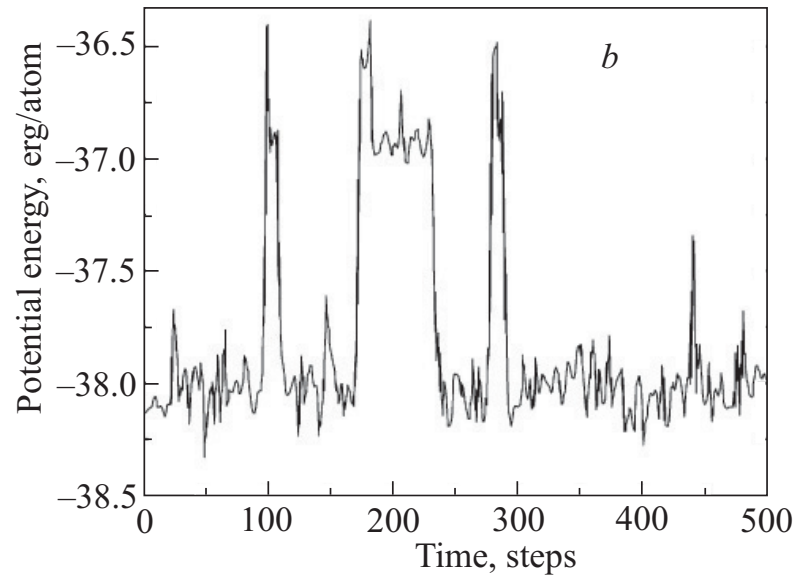

Fig. 4. Time variation of the total potential energy of cluster atoms as a time function, averaged over fluctuations $(a)$ and according to computer simulation [20] for the isolated 13-atom Lennard-Jones cluster at the excitation energy $10.8 D$ that is below the melting energy $(13.8 D)(b)$.

$$
\begin{aligned}
& E_{\text {sol }}=E_{0}+\frac{3 n-6}{2} \cdot \frac{1-\eta_{\text {sol }}}{\eta_{\text {sol }}} T_{\text {sol }}, \\
& E_{\text {liq }}=E_{0}+\Delta E+\frac{3 n-6}{2} \cdot \frac{1-\eta_{\text {liq }}}{\eta_{\text {liq }}} T_{\text {liq }},
\end{aligned}
$$

where $n$ is the number of cluster atoms. In reality, the anharmonicity coefficients are close to $1 / 2$, but their difference is important. The values of the anharmonicity parameter $\eta$ as a temperature function are given in Fig. 5 for the Lennard-Jones cluster.

The energy of configuration excitation of the 13-atom Lennard-Jones cluster does not depend on the temperature in a range of phase coexistence and equals $[15,22,23]$

$$
\Delta E=(2.47 \pm 0.03) D,
$$

where $D$ is the dissociation energy of the Lennard-Jones diatomic.

Let us introduce $w_{\text {sol }}$ and $w_{\text {liq }}$, the probabilities that the cluster be located in the solid and liquid states correspondingly, and under conditions of configuration transitions in a cluster, $w_{\text {sol }}+w_{\text {liq }}=1$. The ratio of these values is expressed through thermodynamic parameters, which under isothermal conditions (a cluster is a canonical atomic ensemble) has the form

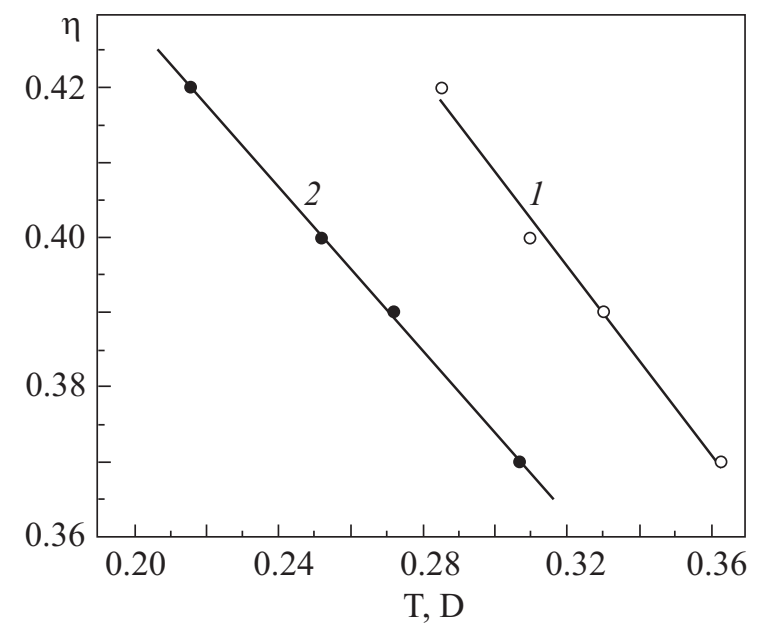

Fig. 5. The anharmonicity parameter for the solid and liquid aggregate states of the isolated 13-atom Lennard-Jones cluster as a function of cluster temperature [15,21] constructed on the basis of the computer simulation data [3] for the solid (1) and liquid (2) aggregate states.

$$
p=\frac{w_{\text {liq }}}{w_{\text {sol }}}=\exp \left(-\frac{\Delta E}{T}+\Delta S\right),
$$

where $\Delta S$ is the entropy change resulting from the phase transition. As is seen, the entropy jump may be determined at a given cluster temperature on the basis of computer cluster simulation with separation the aggregate states. The same operation may be done $[15,21]$ for a cluster as a microcanonical ensemble of atoms with accounting for different atom temperatures for each aggregate state. Below, we analyze the temperature dependence for the entropy jump at the phase transition.

Let us represent the cluster entropy in the form

$$
\Delta S=S_{\text {conf }}+S_{\text {term }},
$$

where $S_{\text {conf }}$ corresponds to configuration cluster excitation, and $S_{\text {term }}$ is determined by a different character of thermal atom motion in the transition states. The first part is for a 13-atom cluster

$$
S_{\text {conf }}=\ln (15 \cdot 12) \simeq 5.2,
$$

where 15 is the number of surface faces that do not border a newly-formed vacancy at configuration excitation, and 12 is the number of final atomic positions from which an atom can be promoted to create the vacancy. This value is independent of the temperature. One can determine the total entropy jump on the basis of dynamic cluster simulations using formula (6). This operation was done on the basis cluster simulations where the 13-atom Lennard-Jones was a microcanonical [3] and canonical [19] ensemble of atoms. The results are reduced to the canonical case and are given in Fig. 6. One can see that the value $S_{\text {term }}$ that follows from formula (7) and Fig. 6, is compa- 


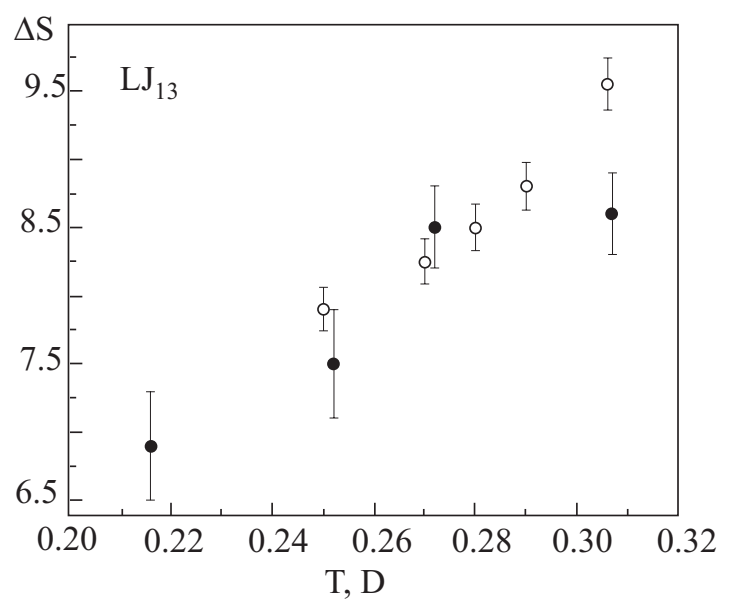

Fig. 6. The entropy jump at melting of the isothermal 13-atom Lennard-Jones cluster. Closed circles are obtained from the results of computer simulation of the isolated 13-atom Lennard-Jones cluster [3], and close circles relate to the isothermal 13-atom Lennard-Jones cluster [19].

rable to $S_{\text {conf }}$ at the melting point. This fact cannot be explained by different Debye temperatures for cluster oscillations with the harmonic oscillations of cluster atoms. One can connect it with transition from locked state of a transferring atom on the cluster surface to a free motion of this atom when the cluster temperature increases. In addition, Table 1 contains some thermodynamic parameters of the 13-atom Lennard-Jones cluster [24,25] obtained on the basis of results of computer simulation [3]. In this table, $E_{\mathrm{ex}}$ is the excitation energy of this cluster as a microcanonical ensemble of atoms, $\Delta T=T_{\text {sol }}-T_{\text {liq }}$ is the temperature difference for aggregate states, other notations are explained for the above formulas.

Table 1. Parameters of the Lennard-Jones cluster (consisting of 13 atoms as a microcanonical ensemble of atoms) following from computer simulation [3]

\begin{tabular}{c|c|c|c|c}
\hline \hline $\mathrm{E}_{\mathrm{ex}} / D$ & 11 & 12.9 & 14.1 & 16.2 \\
$K_{\mathrm{sol}} / D$ & 4.63 & 5.11 & 5.44 & 6.00 \\
$K_{\mathrm{liq}} / D$ & 3.56 & 4.15 & 4.48 & 5.07 \\
$T_{\mathrm{sol}} / D$ & 0.285 & 0.310 & 0.330 & 0.363 \\
$T_{\mathrm{liq}} / D$ & 0.216 & 0.252 & 0.272 & 0.307 \\
$\eta$ & 0.42 & 0.40 & 0.39 & 0.37 \\
$\mathrm{p}$ & 0.1 & 0.4 & 1.8 & 4.0 \\
$\Delta E / D$ & 2.5 & 2.4 & 2.5 & 2.5 \\
$\Delta T / D$ & 0.059 & 0.058 & 0.058 & 0.056 \\
$\Delta S\left(T_{\mathrm{liq}}\right)$ & $6.9 \pm 0.4$ & $7.5 \pm 0.4$ & $8.5 \pm 0.3$ & $8.6 \pm 03$ \\
\hline \hline
\end{tabular}

The peculiarities of metal clusters consist in a large number of excited atom configurations (see Fig. 7) which are close by energy and an additional electron degree of freedom. At low temperatures one can ignore the electron degree of freedom, and then 13-atom metal clusters have the icosahedral structure in the solid state that is separated from configurationally excited states by some energetic gap. Lowest configurationally excited Lennard-Jones and metal clusters consisting of 13 atoms have a different atom configuration. Besides that, an additional cluster excitation in metal clusters opens new atom configurations in contrast to Lennard-Jones clusters. This means that the structure of the liquid state varies in the course of cluster excitation. As a result of these peculiarities of metal clusters, the entropy jump at melting is larger for them then that for Lennard-Jones clusters. Table 2 contains parameters of melting for some metal clusters followed from the results of computer simulation [26]. Note that melting parameters resulted from cluster simulation, depend on the interaction potential taken between cluster atoms. In addition, the computer simulations fulfilled for metal clusters do not separate cluster aggregate states in the range of phase coexistence. Therefore results for metal clusters are less informative and reliable than that for Lennard-Jones clusters. Nevertheless, the above qualitative comparison of metal and Lennard-Jones clusters is valid.
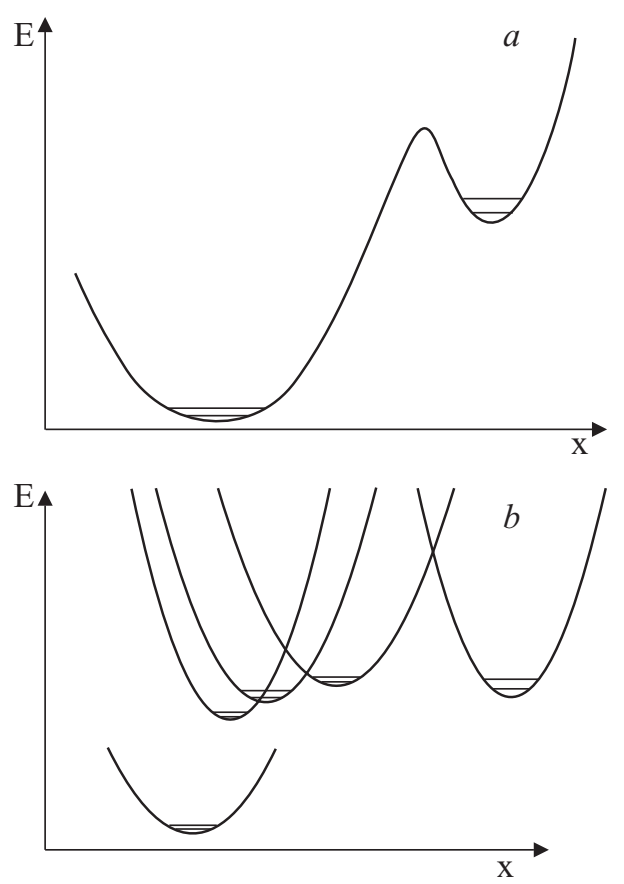

Fig. 7. Projections of PES on a sphere in a many-dimensional coordinate space for a 13-atom cluster of an inert gas $(a)$ and for a 13-atom metal cluster (b). 
Table 2. Parameters of the phase transition for 13-atom metal clusters

\begin{tabular}{c|c|c|c}
\hline \hline Parameter & $\mathrm{Ni}_{13}$ & $\mathrm{Ag}_{13}$ & $\mathrm{Au}_{13}$ \\
\hline \hline$T_{m}, \mathrm{~K}$ & 860 & 420 & 440 \\
$\Delta E, \mathrm{eV}$ & 1.5 & 1.5 & 0.68 \\
$\Delta S_{0}$ & 6.4 & 7.1 & 7.2 \\
$\Delta S_{m}$ & 20 & 41 & 18 \\
$\Delta S_{0} / \Delta S_{m}, \%$ & 32 & 17 & 40 \\
\hline \hline
\end{tabular}

\section{Criteria of cluster melting}

Melting as a phase transition is a collective phenomenon with participation of many atoms, and hence the solid and liquid states differ by the nature of large-scale cooperative behavior of atoms in these aggregate states. In practice, it is convenient to use the Lindemann criterion [27] as a diagnostic for this phase transition. According to this rule, the phase transition occurs at the temperature at which the relative amplitude of atomic oscillations reaches a characteristic value; specifically, melting is said to occur when the ratio of the root-mean-square amplitude of atomic oscillations to the equilibrium distance between nearest neighbors is $0.10-0.15$. Typically, in the range of this temperature, the ratio of distances increases sharply.

Of course, the Lindemann criterion [27] is the simplest criterion to characterize the solid or liquid aggregate state. With the development of numerical methods to simulate cluster dynamics, more precise criteria for the phase transition were introduced, which are based on pair correlations of positions of the cluster atoms. In particular, this correlation function can use the Etters-Kaelberer parameter [28-30] or the Berry parameter [4,31]. These parameters are proportional to the fluctuations of the interatomic distances. These fluctuations give a somewhat more precise insight into how the solid and liquid states differ, and how the transition between them occurs over the range of their coexistence. Among the correlation parameters, the root mean square of the bond length fluctuation $\delta$ is used widely as the melting parameter. It is given by the expression [3]

$$
\delta=\frac{2}{n(n-1)} \sum_{i<j}\left[\frac{\left\langle r_{i j}^{2}\right\rangle-\left\langle r_{i j}\right\rangle^{2}}{\left\langle r_{i j}^{2}\right\rangle}\right]^{2},
$$

where $r_{i j}$ is a distance between atoms $i$ and $j$. For the solid state, in which atoms are fixed in lattice sites, this parameter is typically smaller than 0.1 , less than that for the liquid state with its mobile atoms. This parameter is given in Fig. 8 for the 13-atom Lennard-Jones cluster with the argon pair interaction parameters [3]. One can see that it has

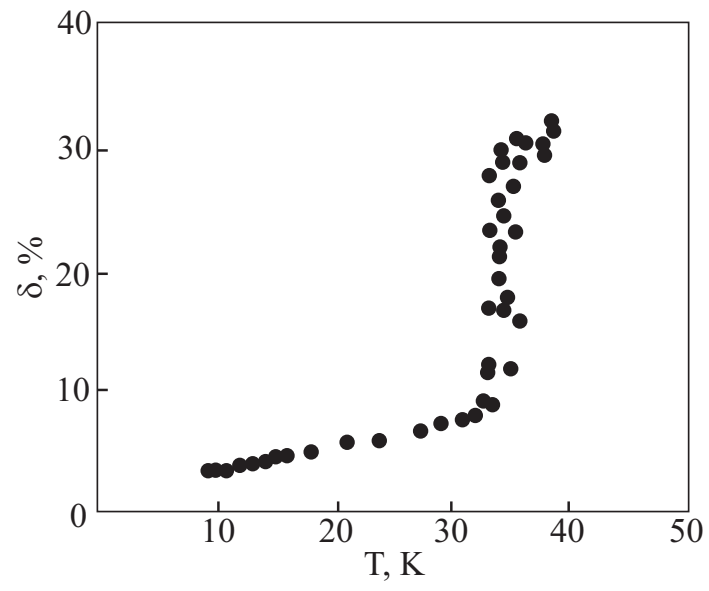

Fig. 8. Averaged over a long period the square length of fluctuations of the relative bond length defined by formula (8) for the 13-atom Lennard-Jones argon cluster [3].

a jump at the melting point, and this jump may be as a definition of the melting point.

We have that the nature of the aggregate states and phase transitions is connected with configuration cluster excitations, while all the above criteria are based on parameters of thermal atom motion. This contradiction is solved by Eq. (7) that exhibits an essential contribution of the difference in thermal motion of cluster atoms in states of transition to the transition entropy. Just this part of the entropy is governed by the above criteria, and because the part $S_{\text {term }}$ is approximately one half of the total transition entropy, the above criteria are valid. Table 3 contains thermodynamic parameters of Lennard-Jones clusters consisting of 13 and 55 atoms, and bulk inert gases [15,25].

Table 3. Parameters of melting for Lennard-Jones (LJ) clusters and macroscopic inert gases

\begin{tabular}{c|c|c|c}
\hline \hline Parameter & $\left.\mathrm{LJ}_{13}^{*}\right)$ & $\left.\mathrm{LJ}_{55}^{*}\right)$ & Bulk inert gases \\
\hline \hline$T_{m} / D$ & 0.29 & 0.31 & 0.58 \\
$\Delta E / D$ & 2.5 & $16 \pm 1$ & $0.98 n$ \\
$\left(T_{\mathrm{sol}}-T_{\mathrm{liq}}\right) / T_{m}$ & 0.22 & $0.31 \pm 0.02$ & 0.56 \\
$\Delta S_{0}$ & 5.2 & $31 \pm 2$ & $0.73 n$ \\
$\Delta S_{m}$ & 8.6 & $48 \pm 4$ & $1.68 n$ \\
$\Delta S_{0} / \Delta S_{m}, \%$ & 60 & $65 \pm 10$ & 44 \\
\hline \hline
\end{tabular}

\section{Cluster phase coexistence}

Dynamic coexistence of cluster phases that takes place near the melting point, results from a restricted entropy jump at melting of small clusters and influences their behavior near the melting point. In analyzing the phase co- 
existence, we take for definiteness that the range of phase coexistence is given by

$$
0.1 \leq p \leq 10,
$$

where $p$ is given by formula (6). Introducing the width of the coexistence range as $\delta T=T_{1}-T_{2}$, where $p\left(T_{2}\right)=0.1$, and $p\left(T_{1}\right)=10$, we obtain

$$
\delta T \approx \frac{5}{\Delta S} .
$$

From this it follows that the phase coexistence for the 13-atom argon cluster with the Lennard-Jones interaction between atoms (the melting point is $T_{m}=37 \mathrm{~K}$ ) proceeds in the range $28-46 \mathrm{~K}$ according to formula (10). In the same manner, we obtain that the phase coexistence in the 55-atom Lennard-Jones cluster with argon parameters $\left(T_{m}=44 \mathrm{~K}\right)$ proceeds in the temperature range $40-48 \mathrm{~K}$. Next, for the nickel cluster consisting of 13 atoms with parameters near the melting point according to the Table 2 data, the phase coexistence takes place in the temperature range $740-980 \mathrm{~K}$. One can see that a range of phase coexistence for metal clusters is narrower than that for dielectric clusters, and for small clusters is wider than that for large clusters. As it follows from these estimations, phase coexistence is of importance for small clusters when a number of cluster atoms is below 100 .

The possibility to extract different aggregate states from dynamic computer simulations is determined by fluctuations of melting parameters. In particular, the fluctuation of the total kinetic energy of cluster atoms is $\Delta E_{\text {kin }} \sim T_{m} \sqrt{n}$, where $n$ is the number of cluster atoms, and the relative fluctuation of this quantity is

$$
\zeta=\frac{\Delta E_{\mathrm{kin}}}{\Delta E} \sim \frac{T_{m} \sqrt{n}}{\Delta E} \sim \frac{\sqrt{n}}{\Delta S} .
$$

For the 13-atom Lennard-Jones cluster this formula gives $\zeta=0.4$, and for the 55-atom Lennard-Jones cluster we have on the basis of this formula $\zeta=0.16$. Seemingly, from this it follows that separation of aggregate states is possible for large clusters, though in a narrow range. In reality, it turns out well for the 55-atom Lennard-Jones cluster [6], where caloric curves are extracted for three aggregate states. For larger clusters different configuration states are overlapped on the time dependence for cluster parameters (e.g., the total kinetic energy of cluster atoms, the total potential energy of atom interaction). The reason is a large number of configuration excitations. For example, for the Lennard-Jones cluster of 55 atoms the liquid aggregate states includes formation 5-7 voids on the cluster surface $[15,21]$. Hence, excitations with formation of a different number of voids are overlapped with each other, as well as with the ground state. This is absent for a 13-atom cluster. Nevertheless, aggregate states of 13-atom metal clusters may be separated.
Dynamic phase coexistence reflects on some cluster properties in the vicinity of the melting point. We consider this for the heat capacity of a cluster that is a microcanonical ensemble of atoms. In this case temperatures are different for the solid $T_{\text {sol }}$ and liquid $T_{\text {liq }}$ aggregate states, but if the cluster is studied during a large time with many transitions between aggregate states, one can introduce the average cluster temperature. In particular, the average kinetic cluster temperature has the form

$$
T=w_{\text {sol }} T_{\text {sol }}+w_{\text {liq }} T_{\text {liq }}
$$

where $w_{\text {sol }}, w_{\text {liq }}$ are the probability of cluster location in the solid and liquid states, correspondingly. Let us construct the caloric curves for the cluster aggregate states in absence of the phase transition by straightforward lines as it is used in Fig. 9. If a cluster is heated starting from low temperatures, its temperature is $T=T_{\text {sol }}$ at low temperatures and $T=T_{\text {liq }}$ at high temperatures. The transition between these temperatures near the phase transition can give «S-form» $[8,34]$ for the caloric curve that gives the negative heat capacity in the vicinity of the melting point. The analysis $[8,15]$ shows the reality of this character of the caloric curve.

Note that the negative cluster capacity near the melting point does not violate general physical principles. Indeed, the heat capacity is expressed through the derivative from the total kinetic energy of cluster atoms, and the negative heat capacity means that an increase of the total energy of cluster atoms induces an increase of configuration excitation owing to a decrease of the total kinetic energy of cluster atoms. In particular, in the case

$$
1>>\frac{T_{\text {sol }}-T_{\text {liq }}}{T_{\text {sol }}}>>\frac{1}{\sqrt{n}},
$$

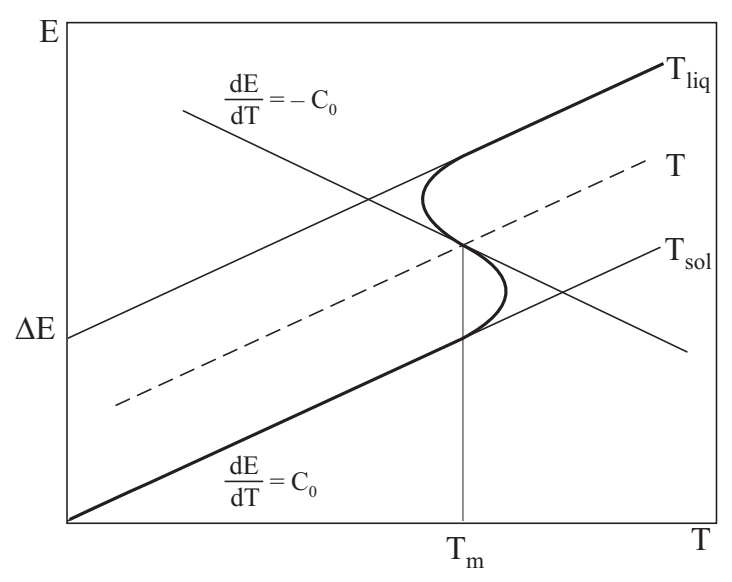

Fig. 9. Caloric curves of an isolated cluster with two aggregate states in the one-temperature approach [35]. 
where $n$ is the number of cluster atoms, the heat capacity at the melting point is $-C_{0}$, where $C_{0}$ is the cluster heat capacity far from the melting point $[15,35]$. The criterion (13) is not fulfilled for the 13-atom Lennard-Jones cluster.

\section{Phase transitions in macroscopic atomic systems}

The concepts developed in the analysis of the cluster phase transitions may be applied to bulk atomic systems, and then they give a more deep understanding of some aspects of this phenomenon compared to a thermodynamic description. Let us show this for condensed atomic systems with a pair interaction. In reality, this corresponds to condensed inert gases, and we below analyze the phase transition in them from the standpoint of the void concept. Note that in contrast to the cluster case, in the analysis of the phase transition in condensed inert gases we will be based on their measured parameters. First of all, we ascertain the character of interaction between atoms that has a pair character because typical values of interaction potentials inside condensed inert gases are small compared the atom ionization potential or compared to other values of the order of an atomic value.

Table 4. Parameters of interaction of two inert gas atoms $\left(R_{e}, D\right)$ and the reduced parameters of the solid aggregate state at the triple point [32]

\begin{tabular}{c|c|c|c|c|c}
\hline \hline Parameter & $\mathrm{Ne}$ & $\mathrm{Ar}$ & $\mathrm{Kr}$ & $\mathrm{Xe}$ & Average \\
\hline \hline$R_{e}, \AA$ & 3.09 & 3.76 & 4.01 & 4.36 & \\
$D, \mathrm{meV}$ & 3.64 & 12.3 & 17.3 & 24.4 & \\
$a / R_{e}$ & 1.02 & 1.00 & 0.99 & 1.01 & $1.005 \pm 0.013$ \\
$T_{\mathrm{tr}} / D$ & 0.581 & 0.587 & 0.578 & 0.570 & $0.579 \pm 0.007$ \\
$\Delta S$ & 1.64 & 1.69 & 1.70 & 1.71 & $1.68 \pm 0.03$ \\
$\Delta H_{\text {fus }} / \Delta S$ & 0.955 & 0.990 & 0.980 & 0.977 & $0.98 \pm 0.02$ \\
$p_{\text {tr }} \Delta V / \Delta H_{\text {fus }}, 10$ & 2.8 & 2.1 & 2.1 & 2.0 & $2.2 \pm 0.4$ \\
$\varepsilon_{\text {sub }} / D$ & 6.1 & 6.5 & 6.5 & 6.5 & $6.4 \pm 0.2$ \\
$\varepsilon_{\text {sol }} / D$ & 6.2 & 6.5 & 6.5 & 6.5 & $6.4 \pm 0.2$ \\
\hline \hline
\end{tabular}

Table 4 contains reduced parameters for solid inert gases near the triple point that are based on the measurable data for parameters of solid inert gases and parameters of the interaction potential of two atoms $\left(R_{e}\right.$ is the equilibrium distance between atoms of the diatomic molecule, $D$ is the depth of the potential well, or the dissociation energy of the classical diatomic molecule). As is seen, the binding energy per atom is closer to the case of a short-range interaction of atoms when interaction between nearest neighbors takes place, rather to the Lennard-Jones interaction. Therefore, in considering

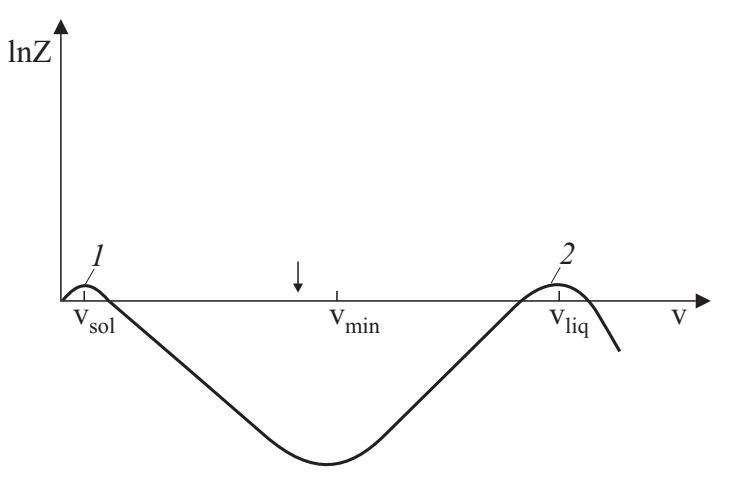

Fig. 10. The logarithm of the partition function for an ensemble of bound atoms with a pairwise atomic interaction as a function of a number of internal voids; 1,2 correspond to the solid and liquid aggregate state, respectively.

condensed inert gases, we restrict by interaction between nearest neighbors.

Applying the void concept to condensed inert gases, we construct the dependence of the specific internal energy $E$ or the specific free energy $F$ (per unit volume) on the specific number of voids $v$ that has the form of Fig. 10 [36] if two aggregate states (solid and liquid) exist.

Note that for condensed inert gases the volume term is approximately three orders of magnitude less than the entropy term near the triple point. Therefore, the internal energy $E$ and the free energy $F$ are equal practically, and we used it. The condition of the second minimum of $F(v)$ and coincidence of the free energies at the first and second minima give equations for $F(v)$ with measured parameters of condensed inert gases at the triple point. These equations exhibit that two aggregate states are possible only in a certain region of energetic parameters. For real condensed gases these equations give the void parameters at the triple point that are given in Table 5 .

Table 5. Void parameters for the liquid aggregate state at the triple point [15]

\begin{tabular}{c|c|c|c|c|c}
\hline \hline Parameter & $\mathrm{Ne}$ & $\mathrm{Ar}$ & $\mathrm{Kr}$ & $\mathrm{Xe}$ & Average \\
\hline \hline$g_{\min }$ & 1.9 & 1.0 & 2.0 & 2.0 & 2.0 \\
$g_{\max }$ & 1900 & 3700 & 4300 & 4100 & $3500 \pm 1000$ \\
$g\left(v_{\text {liq }}\right)$ & 55 & 62 & 63 & 68 & $62 \pm 5$ \\
$v_{\text {liq }} / n$ & 0.320 & 0.319 & 0.318 & 0.319 & $0.319 \pm 0.001$ \\
$\mathrm{~V}_{\text {void }} / V_{\text {sol }}$ & 0.49 & 0.49 & 0.49 & 0.49 & $0.48 \pm 0.02$ \\
$\varepsilon_{\text {liq }} / D$ & 3.00 & 3.09 & 3.05 & 3.05 & $3.05 \pm 0.04$ \\
\hline \hline
\end{tabular}

If we use this operation in a wide range of temperatures, one can construct the dependence of the free energy for a solid and liquid states of condensed inert gases separately, as it is given in Fig. 11 [37] for argon, and the calo- 


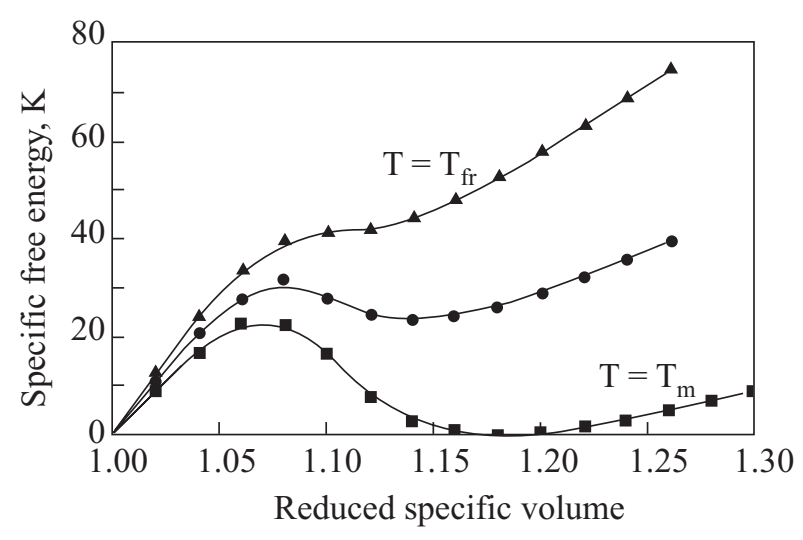

Fig. 11. The dependence of the specific free energy of bulk argon on the volume per atom [37].

ric curve for the liquid aggregate state finishes at the freezing point (Fig. 12). As is seen, the liquid state as the aggregate state does not exist at low temperatures below the freezing point $T_{\mathrm{fr}}$, that, for example, equals to approximately $52 \mathrm{~K}$ for argon (the triple-point temperature is $83.4 \mathrm{~K}$ ). In the case of fast cooling, a glassy state is formed at temperatures below the freezing point. Because of the activation character of decay of this state, it can live at low temperatures very long, but the properties of this condensed atomic system differ from those of the liquid states. Note that the glassy state may be formed in another way, by deposition of atoms on a target of a low temperature, as it was made in experiment [38] for argon with the target temperature of $10 \mathrm{~K}$. Subsequent heating of a formed deposit can lead to formation of the crystal structure, as is shown in Fig. 13.

Let us consider the system of identical repulsed particles, and examples of such systems are given in Table 6 [15]. Below we restrict ourselves mostly by inert gases at high pressures. The repulsed interaction potential of two inert gas atoms can be approximated by the formula

$$
U(R)=U\left(R_{0}\right)\left(\frac{R_{0}}{R}\right)^{k}
$$

and the parameter $k$ of this formula in a range of repulsed potentials $0.1-1 \mathrm{eV}$ varies in limits $6-8$ for atoms of inert gases [39]. Since the condition

Table 6. Ensembles of repulsed particles and boundary conditions which allow one to concentrate the particles in a restricted space region

\begin{tabular}{c|c}
\hline \hline Ensemble of particles & Boundary condition \\
\hline \hline Inert gases under high pressure & External pressure \\
Hard balls in a box & Pressure under weight of upper particles \\
Colloid solution & External pressure \\
Dusty plasma & Electric traps \\
\hline \hline
\end{tabular}

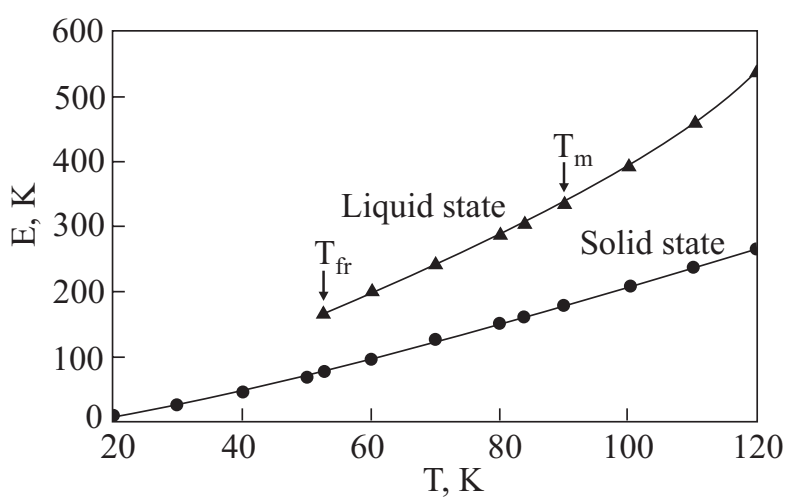

Fig. 12. The caloric curves for bulk argon [37].

$$
k=\frac{d \ln U}{d \ln R}>>1
$$

corresponds to the model of hard balls, we will consider interaction of atoms in condensed inert gases under high pressures within the framework of this model that simplifies the analysis.

It is convenient to characterize the state of the ensemble of repulsed balls by the packing parameter $\varphi[40]$ that is introduced according to the formula

$$
\varphi=\frac{4 \pi n}{3 V} r^{3},
$$

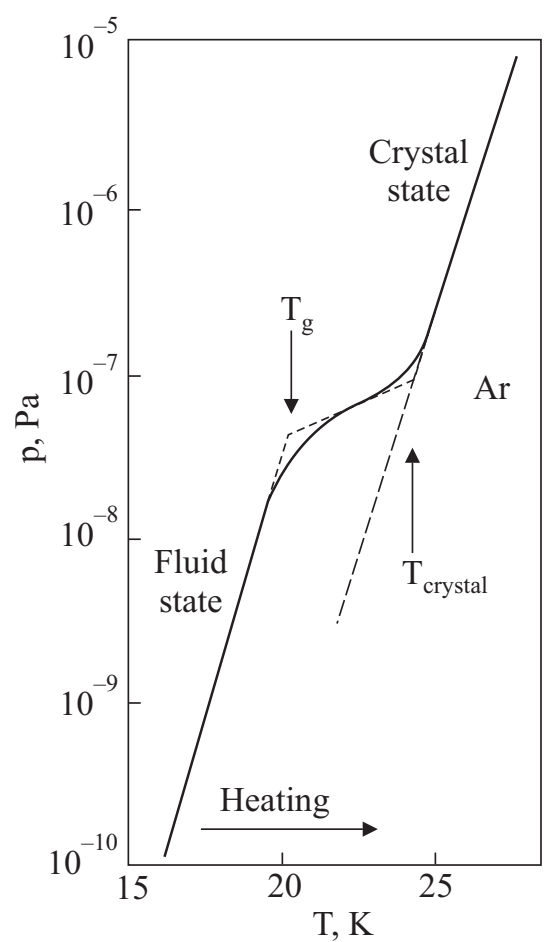

Fig. 13. The temperature dependence of the saturated vapor pressure above a heated amorphous argon formed by deposition of the argon flux on a copper substratum at the temperature $10 \mathrm{~K}$ $[38,40]$ and referred to the glassy-like and crystal argon states. 
where $r$ is a ball radius, $n$ is a number of balls in the ensemble, and $V$ is the volume occupied by this ensemble. In the most dense distribution of balls when they form the crystal lattice of the face-centered cubic or hexagonal structure, the packing parameter is $\varphi=0.74$; in other cases its value is less. According to computer simulation, the packing parameter of an ensemble of identical balls at zero temperature is $\varphi=0.64$. This value follows also from filling of a container with hard balls [41-43]. As is seen, the packing parameter for an infinite ensemble of repelling hard balls is lower than that for a uniform infinite crystal of the close packed structure. This means that particles of this ensemble do not form a crystal lattice even at zero temperature. The same result follows from the virial theorem for particles interacted through the potential (14) with $k>3$ [44], so that the structure of an uniform crystal is unstable.

This unexpected result from the first glance causes additional questions about the structure of an ensemble of repelling particles at zero temperatures. Partially, the answer follows from experiments with colloid suspensions of insoluble particles. For example, in experiment [45] with a size of an individual particle of $0.17 \mu \mathrm{m}$ a particle ensemble consisted of individual crystallites of size of approximately $100 \mu \mathrm{m}$, and each crystallite contained in average $4 \cdot 10^{7}$ monomers. Thus, at zero temperature the system of repulsed particles consists of separate solid clusters (domains) which size depends on the character of interaction.

Condensed inert gases (excluding helium) form a crystal lattice of the face-centered cubic structure at atmospheric pressure and low temperatures. A pressure increase leads to formation of dislocations that results from the stacking instability [46] due to mixing of the face-centered cubic and hexagonal structures. Unfortunately, we do not know the size of individual crystallites in the structure of condensed inert gases at high pressure, as well the dependence of parameters of their structure at high pressure as the pressure increases.

In spite of an expected behavior at low temperatures, the system of repulsed particles has two aggregate states, as well as other simple systems of particles. In particular, the equation of the melting curve for an ensemble of repulsed balls according to computer simulations [47] has the form

$$
\frac{p V_{\text {sol }}}{T}=11,
$$

where $p$ is the pressure, $V_{\text {sol }}$ is the specific volume for the solid state, $T$ is the melting point at this pressure. The reduced volume jump is $\Delta V / V_{\text {sol }}=0.103$, the entropy jump is $\Delta S=1.16$, and $p \Delta V=T \Delta S$ in this case [47].

As a demonstration of the phase transition in inert gases at high pressure, in Fig. 14 we present the argon

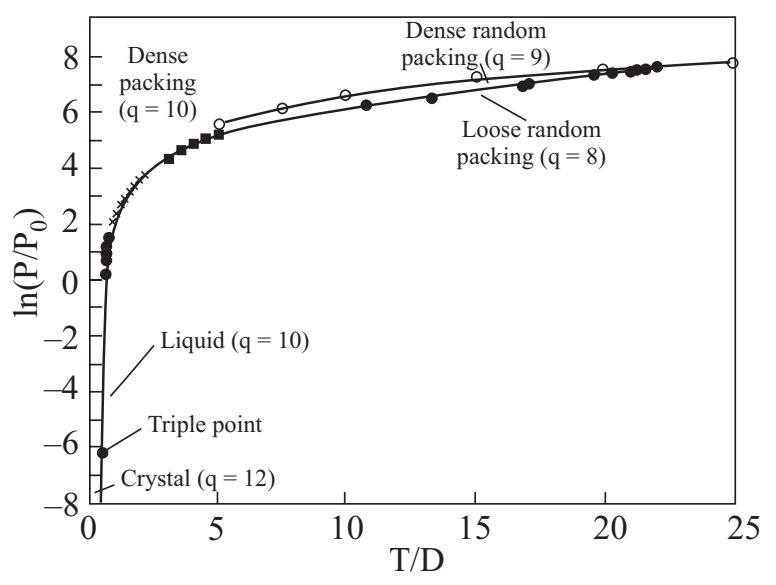

Fig. 14. The melting curve for condensed argon: solid signs are experimental data, open circles are constructed on the basis of the results of computer simulation [47], $q$ is a number of nearest neighbors for an internal atom.

melting curve, where the experimental curve [46] is approximated by the pressure dependence

$$
\frac{d p}{d T}=4+2.1\left[\left(\frac{T}{T_{\text {tr }}}\right)^{0.78}-1\right] .
$$

Here the derivative $d p / d T$ is expressed in $\mathrm{MPa} / \mathrm{K}$, and $T_{\text {tr }}=83.8 \mathrm{~K}$ is the argon triple point.

\section{Conclusion}

The contemporary understanding of the nature of phase transitions in clusters is based in a large degree on cluster computer simulation with methods of molecular dynamics. Combining the results of the dynamic behavior of clusters with small number parameters for their description taken from thermodynamics, we obtain a more deep description of clusters compared to thermodynamic approach within the framework of thermodynamic terms. This leads to some concepts for the phase transitions in clusters which are represented above.

1. J.W. Gibbs, Trans. Conn. Acad. Arts. Sci. 3, 108 (1875); ibid. 3, 343 (1878).

2. J.W. Gibbs, The Collected Works, Longmans and Green, New York (1928).

3. J. Jellinek, T.L. Beck, and R.S. Berry, J. Chem. Phys. 84, 2783 (1986).

4. R.S. Berry, T.L. Beck, H.L. Davis, and J. Jellinek, $A d v$. Chem. Phys. 90, 75 (1988).

5. R.S. Berry, Chem. Rev. 93, 2379 (1993).

6. R.E. Kunz and R.S. Berry, Phys. Rev. E49, 1895 (1994).

7. R.S. Berry, in: Theory of Atomic and Molecular Clusters, J. Jellinek (ed.), Springer, Berlin (1999), p. 1.

8. D.J. Wales, Energy Landscapes, Cambridge University Press (2003). 
9. M.R. Hoare and P. Pal, Adv. Phys. 20, 161 (1971); ibid. 24, 645 (1975).

10. M.R. Hoare, Adv. Chem. Phys. 40, 49 (1979).

11. F.H. Stillinger and T.A. Weber, Phys. Rev. A25, 978 (1982).

12. F.H. Stillinger and T.A. Weber, Phys. Rev. A28, 2408 (1983).

13. D.S. Corti, P.G. Debenedetti, S. Sastry, and F.H. Stillinger, Phys. Rev. E55, 5522 (1997).

14. T. Komatsuzaki and R.S. Berry, J. Chem. Phys. 110, 9160 (1999).

15. B.M. Smirnov and R.S. Berry, Phase Transitions of Simple Systems, Springer, Heidelberg (2007).

16. B. Vekhter, K.D. Ball, J. Rose, and R.S. Berry, J. Chem. Phys. 106, 4644 (1997).

17. H. Reiss, H.L. Frisch, and J.L. Lebowitz, J. Chem. Phys. 31, 369 (1959).

18. D.J. Wales and R.S. Berry, J. Chem. Phys. 92,4283 (1990).

19. H.L. Davies, J. Jellinek, and R.S. Berry, J. Chem. Phys. 86, 6456 (1987).

20. J.D. Honeycutt and H.C. Andersen, J. Phys. Chem. 91, 4950 (1987).

21. R.S. Berry and B.M. Smirnov, JETP 100, 1129 (2005).

22. B.M. Smirnov, Phys. Uspekhi 37, 1079 (1994).

23. B.M. Smirnov, Phys. Scripta 100, 1129 (1994).

24. R.S. Berry and B.M. Smirnov, Phys. Uspekhi 48, 331 (2005).

25. R.S. Berry and B.M. Smirnov, J. Non-Cryst. Sol. 351, 1543 (2005).

26. H. Arslan and M.H. Güven, New J. Phys. 7, 60 (2005).

27. F.A. Lindemann, Z. Phys. 11, 609 (1910).

28. R.D. Etters and J.B. Kaelberer, Phys. Rev. A11, 1068 (1975).

29. R.D. Etters and J.B. Kaelberer, J. Chem. Phys. 66, 5512 (1977).
30. J.B. Kaelberer and R.D. Etters, J. Chem. Phys. 66, 3233 (1977).

31. Y. Zhou, M. Karplus, K.D. Ball, and R.S. Berry, J. Chem. Phys. 116, 2323 (2002).

32. B.M. Smirnov, Principles of Statistical Physics, Wiley VCH, Berlin (2006).

33. T.L. Beck, J. Jellinek, and R.S. Berry, J. Chem. Phys. 87, 545 (1987).

34. M. Bixon and J. Jortner, J. Chem. Phys. 91, 1631 (1989).

35. R.S. Berry and B.M. Smirnov, JETP 98, 366 (2004).

36. B.M. Smirnov, JETP 85, 1010 (1997).

37. R.S. Berry and B.M. Smirnov, JETP 93, 541 (2001).

38. A. Kouchi and T. Kuroda, Jap. J. Appl. Phys. Part 2, 29, L807 (1990).

39. V.B. Leonas, Sov. Phys. Usp. 15, 266 (1972).

40. I. Gutzow and J. Schmelzer, The Vitreous State, Berlin: Springer (1995).

41. J.D. Bernal, Nature 183, 141 (1959).

42. G.D. Scott, Nature 178, 908 (1960).

43. J.D. Bernal and J. Mason, Nature 188, 908 (1964).

44. R.S. Berry and B.M. Smirnov, Phys. Rev. B71, 051510 (2005).

45. P.N. Pusey, W. Van Megen, P. Bartlett, B.J. Ackerson, J.G. Rarity, and S.M. Underwood, Phys. Rev. Lett. 63, 2753 (1989).

46. R. Boehler, M. Ross, P. Soderlind, and D. Boercker, Phys. Rev. Lett. 86, 5731 (2001).

47. W.G. Hoover, S.G. Gray, and K.W. Johnson, J. Chem. Phys. 55, 128 (1971). 